\title{
Federated Accounting Management System Architecture for Multimedia Service Usage Management
}

\author{
B. Bhushan ${ }^{1}$, T. Gringel ${ }^{1}$, C. Ryan ${ }^{2}$, \\ E. Leray ${ }^{2}$, E. de Leastar ${ }^{2}$, and J. Cloney ${ }^{2}$ \\ ${ }^{1}$ Fraunhofer-FOKUS, Kaiserin-Augusta-Allee 31, D-10589 Berlin Germany \\ \{bhushan, gringel\}@fokus.fhg.de \\ ${ }^{2}$ TSSG, Ground Floor Unit A, Waterford Business Park, Cork Road Waterford Ireland \\ \{edeleastar,jcloney, cryan, eleray\}@tssg.wit.ie
}

\begin{abstract}
Service consumers are increasingly becoming aware of QoS and service subscriptions. Service providers (SPs) are also recognizing the opportunity to generate revenue by bundling services in packages and providing them to customers. Standardization bodies have begun to address requirements of charging and usage accounting management as SPs are increasingly relying on them. Federated accounting management concept stems from these developments. It specifically supports the requirement of SPs to co-operate in a federated manner and share the revenue. It also supports the requirements of charging and billing being subcontracted to a third-party. This paper proposes that standard-based interfaces and shared information model, and a wellaccepted development methodology are keys to enabling co-operation between SPs. It presents a requirement analysis and a federated accounting management system architecture, which are based on the recommendations laid down by TMForum, IPDR, OMG and IETF organisations. The architecture supports mediation, rating, charges aggregation, settlement, and online billing.
\end{abstract}

\section{Introduction}

As broadband networks and UMTS infrastructure are more widely deployed, IP-based services are set to become more advanced and will be provided in customized manner. As a result, the demands of e-business are going to drive rapid innovation and SPs are going to provide new services in a B2B (business-to-business) environment. Recent surveys such as [1] and [2] chart these developments in a B2B context. The emergence of the B2B market is also influencing the way services are provided, charged and billed. An important feature in this development is the usage of composite services (several service bundled in one). Composite services consist of several types of services and can be provided by ISPs, virtual private network (VPN) and application service providers (ASP). In this environment, SPs face two important challenges: (1) operating services and maintaining QoS across multiple administrative domains; (2) integrating the charges for various services that make up a composite service. These challenges manifest themselves in a number of ways. For example, new types of B2B interactions have emerged mainly at the levels of service operations

The original version of this chapter was revised: The copyright line was incorrect. This has been corrected. The Erratum to this chapter is available at DOI: 10.1007/978-3-540-45812-8_28

K.C. Almeroth and M. Hasan (Eds.): MMNS 2002, LNCS 2496, pp. 12-24, 2002.

(C) IFIP International Federation for Information Processing 2002 
management. New requirements for customer support, Quality of Service (QoS) maintenance, charge settlement and billing have also emerged [3]. SPs have also acknowledged the emergence of a new stakeholder in the existing business model. The EU-sponsored IST project FORM in which the work presented in this paper was carried out termed this new stakeholder inter-enterprise service provider or IESP.

The IESP can function as a portal to a wide array of services (simple or composite). It can also function as a service retailer or as a specialised service provider who composes new services on customersí demand and deal with the customer queries and bills on behalf of other SPs. This role of IESP adds a new dimension to Billing business model, which is, outsourcing of Billing operations management. Charging and billing is something without which SP businesses will not be commercially viable [4, [5]. Outsourcing Billing is proving to be effective because billing operations are increasingly becoming a specialised and automated task.

The core management operations that SPs require involve customer support, QoS management, charging, billing, and SP settlement [13]. The IESP can play a crucial role in providing them where it deals with customer queries and SLAs (Service Level Agreements) on behalf of SP [6]. It can also do the settlement of the charges amongst SPs if they collectively provided the service and present the bill to the customer. Here one can see three functional areas in operation: (1) customer support and SLA management; (2) QoS management; (3) charging and billing.

\subsection{Related Works}

Organisations such as TMForum (Telemanagement Forum), IPDR (Internet Protocol Detail Record) and IETF, OMG (Object Management Group) and ETSI (European Telecommunication Standards Institute) have come up with guidelines to develop OSS in these three functional areas. The work presented in this paper is relevant to these organisations; hence a brief introduction to their works is given in this section.

TMForum through its work on TOM (Telecom Operations Map) [7] and its successor eTOM [8] has produced a map of operations processes that can be used for the development of three functional areas mentioned above. Within TOM, these functional areas are called $F$ ulfilment, $A$ ssurance and Billing (or $F A B$ ).

The main goals of IPDR organisation are to define an open, flexible record format (the IPDR Schema) for exchanging usage information and essential parameters for any IP transaction [9]. For the specification of interfaces between OSSs (operation support system) and BSSs (business support system), the IPDR organisation has adopted the core functional roles and interfaces that TMFís TOM [7] has identified.

The AAA (Authentication, Authorisation, Accounting) WG of IETF has also produced a set of RFC on accounting management protocol and services [10], [11]. The AAA group can be compared to TOM process flow-through where OSS is regarded as a complete process chain. In the chain Fulfilment feeds relevant information into Assurance, which in turn feeds relevant information into Billing (FAB). FAB themselves roughly correspond to Authentication, Authorisation and Accounting. Of these three broad areas, this paper focuses on the Billing area of TOM or Accounting sub-group of AAA WG.

Having introduced the paper, an outline of the rest of the paper is given here. Section 2 presents the concept of the federated accounting management. Section 3 
describes the business context and presents requirement analysis model. Section 4 presents the federated accounting information model. Section 5 describes the federated accounting system architecture in details. Section 6 outlines the technology used to implement the system architecture. Section 7 concludes this paper and discusses the future work.

\section{Federated Accounting Management Concept}

This section introduces the concept of federated accounting management and briefly describes its main characteristics.

Distribution of Operational Processes and Use of Interdomain Interfaces: Service mediation, rating, and billing processes are distributed over several administrative domains. These processes interoperate by the means of well-defined interfaces. Interfaces may also exist at the boundary between two domains, in which case they are also called Reference Point. The processes mentioned here are already widely known through the TOM map where they are parts of the Billing process [7]. Interfaces between processes have also become known through the work of IPDR Operational Model [9]. However interfaces between domains are not covered in sufficient details by TOM and IPDR and still require further investigation. This paper presents the design of interdomain interfaces (see Sect. 5).

Inter-working of Fulfilment, Assurance and Billing Processes: These three processes take part in so-called end-to-end process flow. Although the Fulfilment and Assurance are separate functional areas in their own right, they co-operate with the Billing process. Federated accounting management incorporates this end-to-end process flow where Fulfilment and Assurance provide Billing with SLA and QoS details. This enables the service provider to apply different tariffs for different types of subscriptions or QoS provided.

A More Inclusive View of Accounting Information: Federated accounting management holds a more inclusive view in which usage information and charges details together form a part of accounting information. IPDR organisation is working on a standardised information model. However, the work of IPDR is confined to producing specification of usage information exchanged. The concept of federated accounting management complements the work of IPDR organisation by providing rationale for and specification of charge details as a part of federated accounting information model (see Sect. 4).

Use of Differential Tariffs: Charging regime uses tariffs that vary with customer types, QoS or any other factor that SPs choose to use. An earlier work by authors on this can be found in [12].

Charge Aggregation and Payment Settlement: In federated accounting management several third-party providers collectively provide their services. IESP acts on behalf of both customer and third-party providers. It aggregates charges and invoices the customer for all the services used; a single invoice is sent to the customer. IESP may also do the settlement for all third-party providers. Work on supplier-provider settlement is currently being addressed in eTOM [8]. 


\section{Business Context and Functional Requirements Analysis}

This section presents the business context, actors involved in federated accounting management and the roles they play (see Fig. 1). The information exchanged between various roles is also shown. This is followed by a description of operations processes involved in federated accounting management. Thereafter key functional requirements that the architecture implements are presented as a use case diagram.

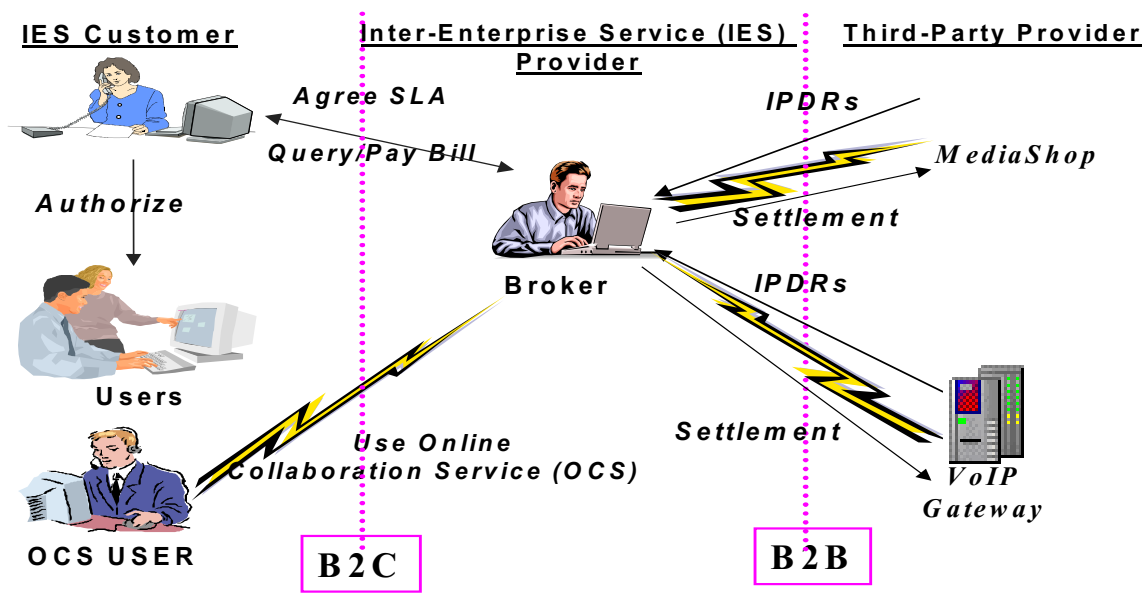

Fig. 1. Federated Accounting Management Business Model

In the business context, the IESP ensures that the customer (or IES Customer in Fig. 1) has access to a variety of services that it needs. They are provided by many SPs (third-party provider in the Fig. 1), who are IESPís partners. OCS (online collaboration service) is one such service, which packages MediaShop and VoIP services in single application. MediaShop is multimedia contents distribution service.

The IES Customer is the subscriber of IES and negotiates and signs a SLA with the IES Provider. It can be a business (organisation) or a private customer. It receives and pays bills for the services used. End-User is a person who uses Inter-Enterprise Service. End-user may be a private user or he/she may work for the IES Customer.

The IESP, as the customer-facing SP, is responsible for SLA handling, tariff negotiation, customer care and accounting aspects of the services offered to the customer by the IESPís partners in a value chain. It provides the IES Customer with access to communication, application, and information services, which are in turn provided by third-party providers (i.e., IRS Provider). IES Provider performs the task of charging and billing of service usage and charge settlement among IES Customers and third-party SPs. IES Provider acts as a service retailer and maintains contracts with third-party providers and does the final payment settlement.

The IRS (Information Retrieval Service) Provider is a third-party provider that provides its services to IES Customer. The third-party providers must be able to charge the IES Customer for service usage, details of which are sent to the IESP as IPDR documents (also shown Fig. 1). IESP incorporates the various IPDR documents by means of aggregation and rating processes and provides a consolidated bill to the 
IES Customer. In order for interaction to occur between the IESP and third-party providers, IPDR Master schema and specifications are used as a shared information schema and a set of interfaces that all parties support.

\subsection{Operations Processes Involved in Federated Accounting Management}

Fig. 2 illustrates the federated accounting management operations processes in the context of the FAB of TOM [7]. Fig. 2 is based on an original version of FAB figure [7]; various aspects of federated accounting management have been added to original FAB figure and illustrated in Fig. 2. An enhanced version of IPDR XML Schema [9] is used to model accounting information (or E-IPDRs shown in Fig. 2) that is exchanged between various business processes. Sect. 4 discusses the enhancement done to Master IPDR Schema.

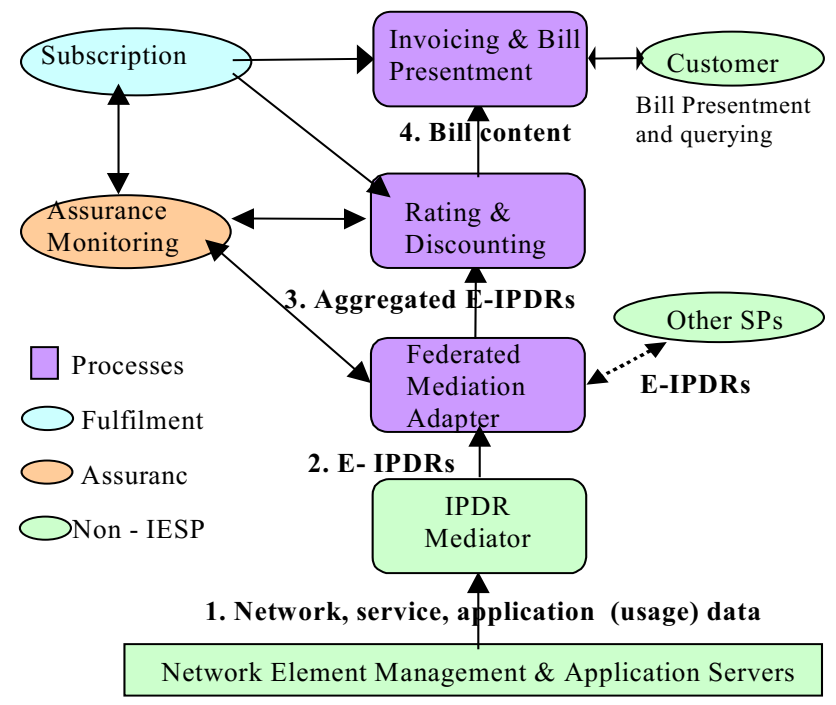

Fig. 2. Federated Accounting Management Operations Process

\subsection{Functional Requirements and Use Case Model}

A comprehensive list of functional requirements is discussed in [12]. The authors have worked on those requirements and narrowed them down to a set of requirements that must be addressed in the design. The narrowed set includes the following requirements and the use case diagram (see Fig. 3) models them:

1. Usage-based charging and real-time response levels.

2. Support for convergence of services (e.g., voice and data).

3. Adaptable federated service mediation facility.

4. Support for a variety of OSS and service value chain. 
5. Charge detail aggregation of composite services.

6. Automated inter SP domain accounting and settlement.

7. Interaction with legacy billing systems.

8. Increased demand for guaranteed QoS and related discounting.

9. Rapid service deployment.

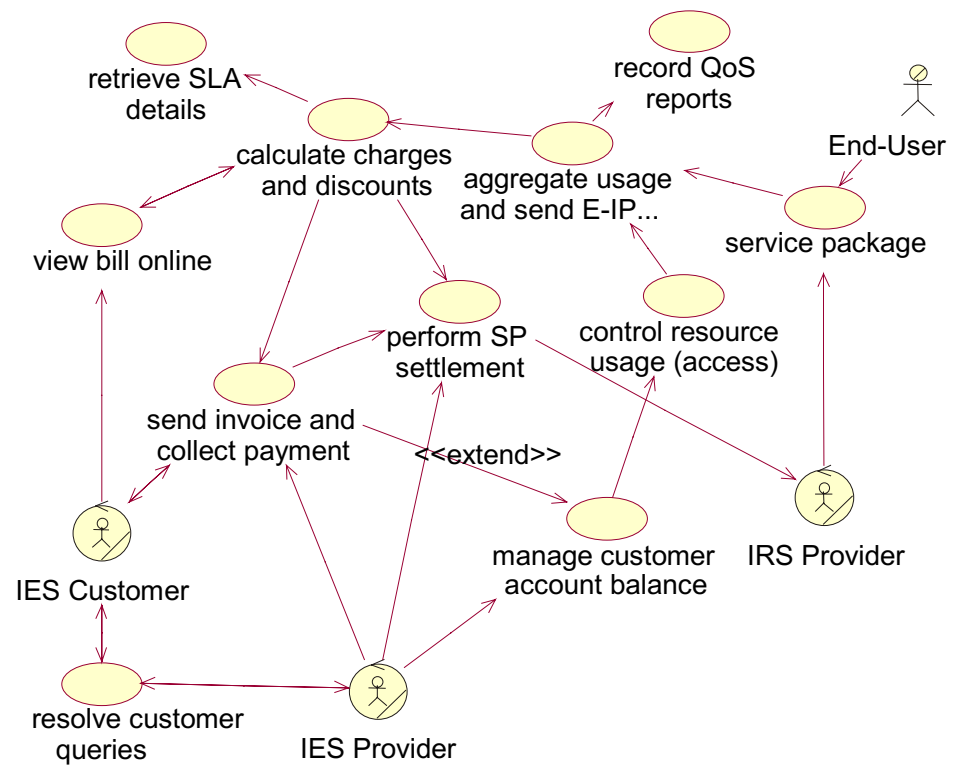

Fig. 3. Federated Accounting Management Use Case Model

Up to this point, this paper has presented the business context and key requirement. The following sections present the information model and design of the accounting management system.

\section{Federated Accounting Information Model}

This section presents the federated accounting information model. Before providing the details of the model, a brief rationale for developing it is given. If accounting management facilities are to be distributed then the SPs must use an information model that: (1) adapts easily to new services; (2) maintains consistency of information during exchange; (3) helps SP in using the information generated by other SPs with little or no effort. Clearly, a common structure, semantic, and syntax of information is crucial in a successful acceptance and functioning of federated accounting management and this is where the federated information model comes into play. 


\subsection{Enhancements to IPDR Master Schema}

The governing idea behind federated accounting management is to support distribution of service mediation and rating facilities across several SP domains. A note for those readers who are familiar with FAB of TMForum, this support means enabling accounting management at BSS level. This is the level at which rates are applied, charges calculated and bills presented.

This is required for the aggregation of charges when a service package is provided to the customer. This package is an agreement that allows the customer to use many services. Several SPs may be involved in providing the package. Clearly SPs have a stake in the bill payments that IESP receives from customer. SPs themselves may want to calculate charges for the services that they contribute towards the service package. IESPís role in this scenario is to aggregate charges and do SP payment settlement. Therefore SPs require means by which they can exchange usage data as well as charges in a single IPDR document. This requirement means that information model must be able to support exchange of usage data as well as charge details.

In order to meet this requirement, this paper proposes federated accounting information model. The model is an enhancement on Master IPDR Schema [9] with a CE (charge entry) has been added to the latter. This enhancement enables IESP to the charge aggregation and settlement in a multi-SPs environment.

The CE holds information such as the charge that the customer owes to SP, discounts provided by the SP, etc. These details are received by IESP and used during SP settlement and payment. If several SPs send their charge details, all charges are aggregated and user pays a single bill for a service package.

The Master Schema that the IPDR organisation has produced does not provide for such requirements because it is outside the scope of the organisation. The federated accounting information model, which is based on Master IDPR Schema, does provide for such requirement. SPs can use CE (charge entry) E-IPDR document (E stands for enhanced) to include charge details and transfer them with IESP.

\subsection{Federated Accounting Information Service-Specific Using UML Notations}

It must be noted that IPDR information model and all model derived from it are implemented using XML Schema Recommendation. This essentially gives us three schemas, which follow from each other in this order: (1) Master IPDR Schema; (2) Federated Accounting Information Schema; (3) Federated Accounting Servicespecific Schema.

Master IPDR Schema: This is the output of IPDR organisation and the basis for all service-specific schemas and federated accounting information schema. This schema has a service-specific part, which can be adapted to any service. SPs can use this schema and adapt its service-specific part to any service for which usage mediation is required. Since federated accounting information schema is based on Master IPDR schema, it is worthwhile to mention it here. For a fuller description, please refer to IPDR specification [9].

Federated Accounting Information Schema: This schema is essentially the Master IPDR Schema, plus the CE element. The CE element is optional and gets populated when SP wishes to calculate its own charges, leaving charge aggregation to IESP. 
Federated Accounting Service-Specific Schema (see Fig. 4): This schema inherits all the elements of Federated Accounting Information Schema, including CE. It is adapted to provide for and record usage events that are specific to a service. For the development of federated accounting management system, two service-specific schemas have been developed; they are for MediaShop (shown in Fig. 4) and VoIP service. Usage attribute specific to these two services are added to the ones that are inherited from federated accounting information schema. The usage information forms the content of E-IPDR Document.

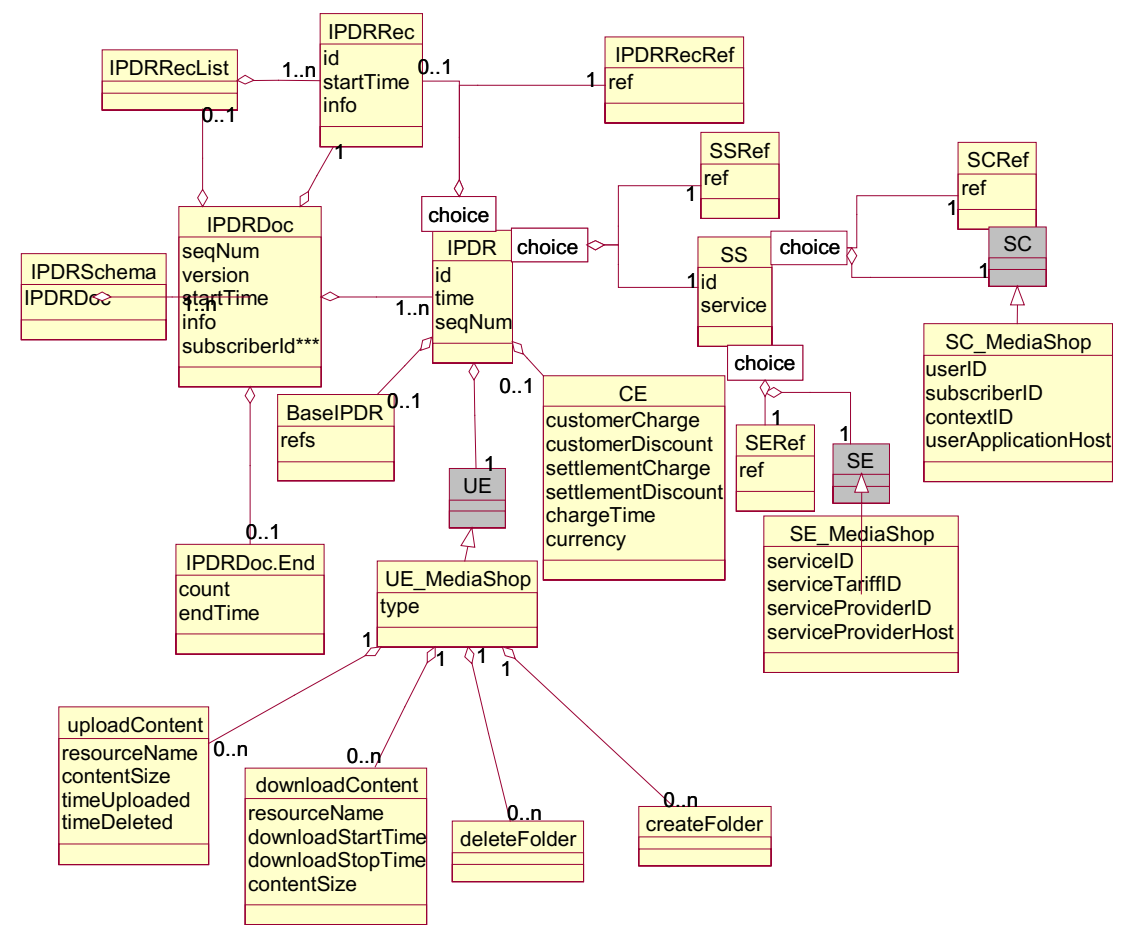

Fig. 4. Federated Accounting Service-Specific Schema for MediaShop Service

Fig. 4 serves three purposes. Firstly, it illustrates how Master IPDR Schema has been used to implement federated accounting information model. One can see the position and contents of CE element in the Fig. 4. Secondly, it also illustrates how federated accounting information model can be specialised to a particular service or network. It also shows how usage information generated by MediaShop can be modelled. Finally, to best of authorsí knowledge, no UML diagram of IPDR Master Schema exists. Therefore the Fig. 4 allows UML users to see how various XML elements of IPDR Schema fit together to form a complete E-IPDR document.

As the reader will see in the next section, federated accounting service-specific schema is an integral part of the system architecture and E-IPDR documents are the information objects communicated at the interfaces such as interdomainAcctMgmt, Rating Bureau Service Interface. All E-IPDR documents are XML instance 
documents. Information objects (mainly usage and charge details) are integrated under a single tree-shape structure. Integration is done at the level of federated accounting management system boundary. Objects and structure are shared by all system interfaces, a shared information schema.

\section{System Architecture}

This section provides the definitions of components and interfaces of the federated accounting management systems architecture. The architecture is specified using UML (Unified Modelling Language) therefore a mapping of the components and interfaces onto to UML artefacts, namely boundary class, control class and information object is also briefly discussed (see Fig. 5).

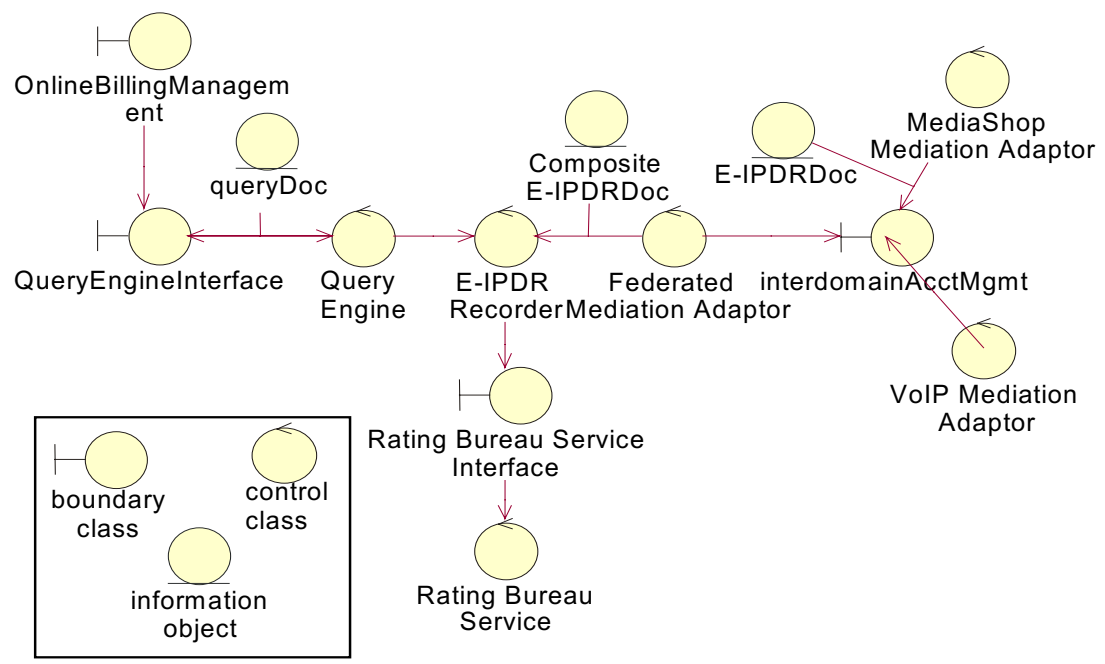

Fig. 5. Components of Federated Accounting Management System Architecture

An interface can be considered a boundary class. A component itself can be considered a control class. The term boundary has a strong connotation of something facing outside or an interface. Among other matters, an interface deals with communication protocols. If communication protocol changes, we need to change the interface; we need not change the component that offers the interface.

The term control class has a strong connotation of something that receives things and manipulates them without paying much attention to how they will be sent to other components. The things that control objects manipulate are information objects. A component deals with co-ordination task, leaving communication task to its interface. A component can have its own state, change its state independently of other components, and can send external event whenever it wishes to do. If co-ordination task changes we may need to change the component but we can still use the same interface offered by the component. 
It co-ordination work is not complicated and sizeable enough then we may not need control object at all. A boundary object will be enough to do communication as well co-ordination.

\subsection{Interfaces (Boundary Objects)}

InterdomainAcctMgmt: This interface resides between IESP and third-party providers and supports usage mediation in a federated SPs environment. This interface is provided by the Federated Mediation Adaptor (FMA) component. Mediation Adaptors (MAs) can use this interface to send E-IPDR documents to FMA. OnlineBillingManagement: This interface resides between IESP and IES Customer. This is the customer entry point to the charging information stored by the IESP.

\subsection{Components (Control Objects)}

Federated Mediation Adaptor (FMA): This component supports collection and aggregation of E-IPDR documents and generation of a composite E-IPDR document. The documents are aggregated under a single usage session (or parent session), which denotes the beginning and end of service package use.

MediaShop Mediation Adaptor (MA): MediaShop MA collects usage data, records the usage events, generates an E-IPDR document, and sends it to FMA.

VoIP Mediation Adaptor (MA): The main role of VoIP MA is to collect usage data, record the usage events, generate an E-IPDR document, and send it to FMA.

QueryEngine: It ensures the availability of all recorded IPDR information.

E-IPDR Recorder: The IPDR Recorder takes upon the role of IPDR documents recorder/transmitter. It implements method specifies in the IPDR ì Protocol Primitives and Parametersî specification (Push, Pull, Subscribe, etc).

Rating Bureau Service (RBS): RBS applies tariffs, calculates discounts and calculates charges for individual services that make up a composite service, for example, OCS (i.e., Online Collaboration Service mentioned in Sect. 3). Then all individual charges are aggregated into a single composite service charge. Finally, RBS generates and stores a rated/discounted E-IPDR document.

\subsection{Information Objects (E-IPDR Documents)}

Composite E-IPDR Doc: This is a list of E-IPDR documents, each of which is an instance document of federated accounting service-specific schema. Its main role is to carry usage information detailing usage of a composite service, as opposed to usage information for a singleton or simple service.

QueryDoc: An XML instance document, containing end-userís query.

E-IPDR Doc: This is single E-IPDR document, based on federated accounting service-specific schema. Its main role is to carry usage information for a singleton service. This information is details of usage events that are generated when the enduser uses MediaShop or VoIP services. 


\section{System Integration and Technology Architecture}

This section discusses how a prototype federated accounting management system was implemented. Fig. 6 shows the technologies that have been used to implement and integrate components of the prototype.

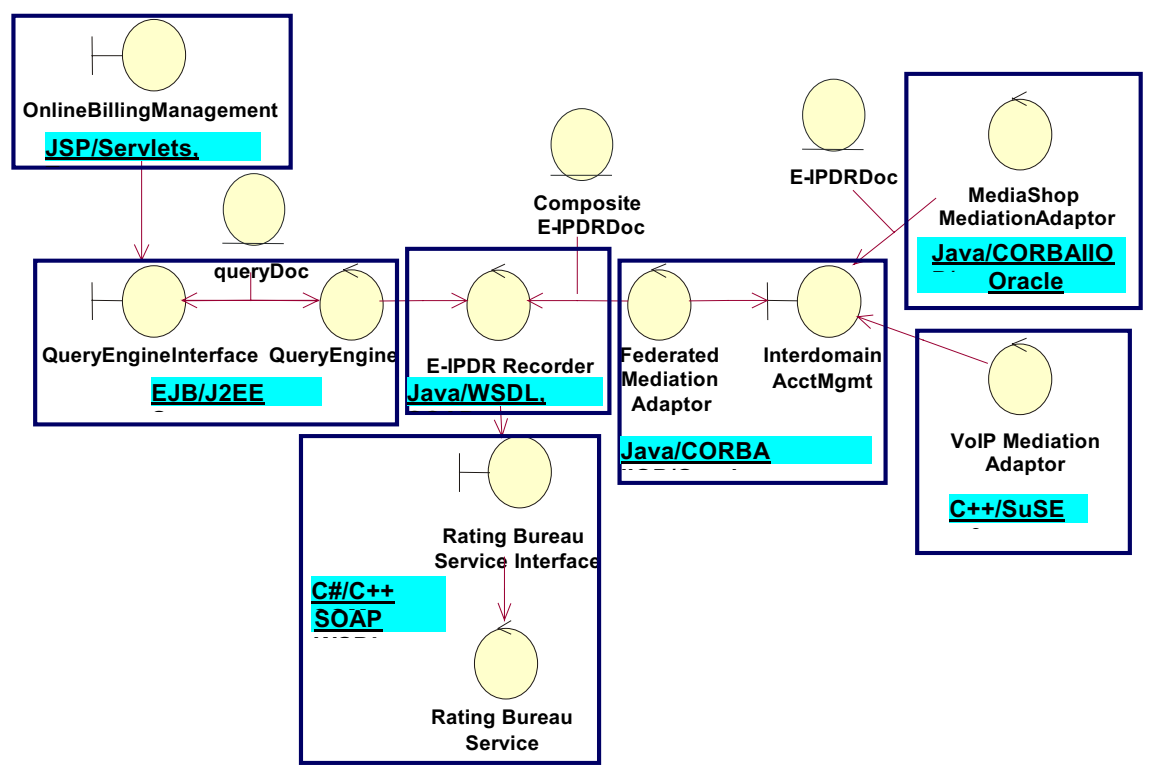

Fig. 6. Federated Accounting Technology Architecture

The main components (see Sect. 5.2) of the prototype were implemented using $\mathrm{C}++$, Java, and C\# and used IIOP, RPC COM/CORBA-SOAP for communication with one another. Interfaces were specified using CORBA IDL and WSDL.

Information objects (or E-IPDR and Composite E-IPDR documents) exchanged between components are XML instance document. They are modelled on federated accounting service-specific schema, which is specified using W3C XML Schema, Structure and Data Types. Oracle is used as storage for E-IPDR documents.

The technology described here has yielded many benefits. Java and EJB make architecture components highly portable. XML/WSDL allows service providers to deploy accounting components as Web-services and seamlessly exchange accounting information. Oracle enables direct mapping of IPDR structure (XML documents) onto database tables. SOAP binds IPDR documents and HTTP tightly, making document exchange them over the internet easy. OSP (Open Service Platform) is a service subscription and access management middleware, which is being developed by Fraunhofer-FOKUS. It is based on TSAS (Telecommunications Service Access and Subscription).

A number of lessons were learnt from the development and evaluation of the federated accounting system. Some important lessons are discussed here.

An interface at the boundary between two SP may possess more functionality then assigned during design phase. For example, interdomainAcctMgmt interface may also 
monitoring and recording network QoS deterioration. Interfaces implementations are kept simple because of the more complex structures (defined in XML) that are passed.

Standardised information model (i.e., IPDR Master Schema) proved to be useful in developing federated accounting service-specific schema. It also reconciles two differing sets of concerns (information-wise), service mediation and charge details that lead to a common goal, i.e., Billing.

There are trade-offs when using XML schemas, which are more complicated than DTDs (Document Type Definitions) but are richer and useful in expressing a vocabulary of federated accounting management business process.

\section{Conclusions and Future Work}

This paper has presented a requirement analysis model and architecture of federated accounting management system. The system presented has been developed and tested and evaluated in two trials. In order to ensure that components and interfaces provided the required functionality, test case result were mapped onto requirement captured in the requirement analysis phase. Mapping resulted in a set of key requirements that were important for the development of federated accounting management system. They are listed in Sect. 3.

One of the prime aims of the research and development was to validate the specification being developed in standardization community and feed the result into standardization process. It is fair to say that authors have successfully achieved this aim. Results obtained have been provided as contributions to a number of international organizations. A significant proportion of design specification has been provided as input to Telecom Domain Task Force of OMG, which is also developing a specification of federated charging and rating facility.

An initial business model and use cases have been provided to AAA WG of IRTF (Internet Research Task Force). A contribution containing intermediate business model and informational model has been provided to TMForum through a TM World conference. There are further plans to provide an enhanced IPDR Master Schema as an input to IPDR organization.

Several areas in which future work can be carried out have identified. Authors consider the use of XML and Webservice technologies (e.g., XSLT, WSDL, SOAP) for component and interface definitions an important area for future work. Therefore further research into Web service technologies will be carried out.

Use of XMI and XSLT in transforming technology-neutral system architecture to a technology-specific system is essential if federated accounting management is to appeal to E-commerce. Another area, which is gaining acceptance, is ebXML. It will be used for the definition of federated accounting management business process.

Further development will also be carried out into a generic approach to aggregated services mediation. In this generic approach, functionality will be added to FMA component, thus making them adaptable to several different types of communication protocol and technologies. Components and interfaces will be further enhanced to support QoS and a guaranteed delivery of E-IPDR documents.

Last but not the least, the authors would like to thank the members of PLATIN group (Fraunhofer-FOKUS Germany) and TSSG group (WIT Ireland) for their 
constructive comments. This research work was carried out under the auspices of EUsponsored IST project FORM (IST-1999-10357). More information on FORM can be obtained from $\mathrm{Nww}$.ist-form.org or from the authors.

\section{References}

1. Varshney, et al, ì Voice Over IPî, Communications of ACM, Jan 2002,Vol 45, No 1.

2. Bartholome, Lloyd, et al, ì A Practical Approach For Implementing E-Commerce Programs in Business Schoolî, Communications of ACM, January 2002, Vol. 45, No. 1.

3. Howard-Healy, ì Billing the future of contentî, Billing, Issue 7, September/October 2000.

4. Browne, ì Have you got money to burn?î, Telecommunications International, Aug 2001.

5. Whitworth, ì The Unknown World of $3 \mathrm{G}$ Tariffsî Telecommunications International, Feb 2001.

6. Dobson ì The SLA Battlegroundî, Telecommunications International, March 2002.

7. Telecom Operations Map, Approved Version 2.1, TeleManagementForum, March 2000.

8. TMForum GB921: ì eTOM: The Business Process Framework For The Information and Communication Services Industryî, Members Evaluation Version 2.0, October 2001.

9. Network Data Management ñ Usage (NDM-U): For IP-based Service, Version 2.6, October 2001, IPDR Organisation.

10. RFC 2924: Accounting Attributes and Record Format, Category: Informational, Authors: N. Brownlee, A. Blount, September 2000.

11. RFC 3127: Authentication, Authorization, and Accounting: Protocol Evaluation, Category: Informational, Authors: Mitton, Johns, Barkley, Nelson, Patil, June 2001.

12. Bharat Bhushan, et al, ì Federated Accounting: Service Charging and Billing in a Businessto-Business Environmentî, IM2001: 2001 IEEE/IFIP International Symposium on Integrated Network Management Proceedings, Seattle, May 2001.

13. Huston, Geoff, ì Interconnection, Peering and Settlements: Part-Iî, The Internet Protocol Journal, Volume 2, Number 1, March 1999. 